Pacific

Journal of

Mathematics

\title{
POSTNIKOV TOWERS AND GOTTLIEB GROUPS OF
} ORBIT SPACES

\author{
Marek Golasiński and Daciberg Lima Gonçalves
}

Volume $197 \quad$ No. 2

February 2001 


\title{
POSTNIKOV TOWERS AND GOTTLIEB GROUPS OF ORBIT SPACES
}

\author{
Marek Golasiński and Daciberg Lima Gonçalves
}

Let $X$ be a 1-connected space with the homotopy type of a $C W$-space and $H$ a finite group acting freely on $X$ by homeomorphisms homotopic to the identity. We prove that $l_{k} \eta_{*} G_{k}(X) \subseteq G_{k}(X / H)$ for all $k>1$ and some estimated positive integer $l_{k}$ which depends on $k$, where $G_{k}$ is the $k^{\prime}$ th Gottlieb group and $\eta: X \rightarrow X / H$ is the quotient map to the orbit space $X / H$. We show that $l_{k}$ is independent of $k$ for $X$ with the homotopy type of a finite $C W$-space. We also obtain that $l \pi_{k}(X) \subseteq G_{k}(X)$ for some positive integer $l$ (independent on $k$ ) provided some restrictions are placed on the space $X$ and the integer $k>1$. Moreover, $\eta_{*} G_{k}(X)_{p}=G_{k}(X / H)_{p}$ for the $p$-primary components, where $p$ is a prime not dividing the order $|\boldsymbol{H}|$ of the group $H$.

\section{Introduction.}

The Gottlieb groups $G_{k}(X)$ of a space $X$ have been defined by Gottlieb in $[\mathbf{2}, \mathbf{3}]$; first $G_{1}(X)$ and then $G_{k}(X)$ for all $k \geq 1$. In the paper [3] it is shown that $G_{2 n}\left(\mathbb{S}^{2 n}\right)=0$ and

$$
G_{2 n+1}\left(\mathbb{S}^{2 n+1}\right)= \begin{cases}\mathbb{Z}, & \text { if } n=0,1,3, \\ 2 \mathbb{Z}, & \text { for any other } n,\end{cases}
$$

where $\mathbb{S}^{m}$ is the $m$-dimensional sphere for $m \geq 1$. On the other hand, by $[\mathbf{1}, \mathbf{1 0}]$ the $p$-localization of an odd dimensional sphere $\mathbb{S}^{2 n+1}$ with $p$ being an odd prime has the homotopy type of an $H$-space. Thus, in view of the result in $[\mathbf{6}]$, we can easily deduce that the $p$-primary component of the Gottlieb group $G_{k}\left(\mathbb{S}^{2 n+1}\right)$ is isomorphic to the $p$-primary component of the homotopy group $\pi_{k}\left(\mathbb{S}^{2 n+1}\right)$ for all $k \geq 1$. Lang [5] developed techniques for calculating Gottlieb groups for some orbit spaces. If a finite group $H$ acts freely on an odd dimensional sphere $\mathbb{S}^{2 n+1}$ then Oprea has shown [7] that the Gottlieb group $G_{1}\left(\mathbb{S}^{2 n+1} / H\right)=\mathfrak{Z}(H)$, where $\mathbb{S}^{2 n+1} / H$ is the orbit space of $\mathbb{S}^{2 n+1}$ with respect to the action of the group $H$ and $\mathfrak{Z}(H)$ denotes the center of $H$. Then Pak and Woo [8] considered the generalized lens spaces 
$L_{2 n+1}(p)$ to prove that

$$
G_{2 n+1}\left(L_{2 n+1}(p)\right)= \begin{cases}\mathbb{Z}, & \text { if } n=0,1,3, \\ 2 \mathbb{Z}, & \text { for any other } n .\end{cases}
$$

This paper grew out of our attempt to extend these results mainly by means of Postnikov tower arguments used in [4] for studying Gottlieb groups. Let $X$ be a 1-connected space $X$ of finite type with a finite group $H$ acting freely on $X$. Then we can search for a relationship between the Gottlieb groups $G_{k}(X)$ and $G_{k}(X / H)$ for all $k>1$. It follows from [3, Theorem 6-2] that $\eta_{*} G_{k}(X) \supseteq G_{k}(X / H)$ for all $k>1$, where $\eta: X \rightarrow X / H$ is the quotient map, and that is the starting point for our further discussion. The main purpose of the paper is finding conditions for the converse inclusion. We present two types of results. The first type of results says that for every prime $p$ not dividing the order $|H|$ of the group $H$ we have $G_{k}(X / H)_{p}=$ $\eta_{*} G_{k}(X)_{p}$ for the $p$-primary components of the Gottlieb groups, where $k>1$. The second type of the result says that there is a positive integer $l$ such that $l \eta_{*} G_{k}(X) \subseteq G_{k}(X / H)$. This number $l$ is given as a product of primes (with multiplicity) which divide the order $|H|$ of the group $H$.

Section 1 serves as an introduction to the rest of the paper. We compare the Gottlieb groups of two spaces $X$ and $X / H$ in terms of their Postnikov towers. Then we deduce in Theorem 1.3 that for any $k>1$ there is a positive integer $l$ such that $l \pi_{k}(X) \subseteq G_{k}(X)$ for all $k>1$ provided there are some restrictions on the space $X$.

In Section 2 we present our main result stated in Theorem 2.3: Let $X$ be a space with a free action of a finite group $H$. Then:

(1) for each $k>1$ there is a positive integer $l_{k}$ such that $l_{k} \eta_{*} G_{k}(X) \subseteq$ $G_{k}(X / H)$;

(2) if $p$ is a prime not dividing the order $|H|$ of the group $H$, then $\eta_{*} G_{k}(X)_{p}=G_{k}(X / H)_{p}$ for the p-primary components.

The results and methods of the first two sections are applied in Section 3 to calculate or estimate Gottlieb groups of orbit spaces of spheres and projective spaces.

The authors are extremely grateful to the referee for helpful suggestions.

\section{Gottlieb groups and Postnikov towers.}

Throughout this paper spaces will be connected, with a base point and of the homotopy type of $C W$-spaces. The $k^{\prime}$ th Gottlieb group $G_{k}(X)$ of a space $X$ defined in $[2,3]$ for $k \geq 1$ is the subgroup of the $k^{\prime}$ th homotopy group $\pi_{k}(X)$ containing all elements which can be represented by a map $\alpha: \mathbb{S}^{k} \rightarrow X$ such that $\alpha \vee \operatorname{id}_{X}: \mathbb{S}^{k} \vee X \rightarrow X$ extends (up to homotopy) to a map $F: \mathbb{S}^{k} \times X \rightarrow X$. The map $F: \mathbb{S}^{k} \times X \rightarrow X$ is called an associated map for $\alpha: \mathbb{S}^{k} \rightarrow X$. If $X^{X}$ is the space of all maps from $X$ to $X$ and 
ev : $X^{X} \rightarrow X$ is the evaluation map at the base point then it can be shown that $G_{k}(X)=\operatorname{Im}\left(\mathrm{ev}_{*}: \pi_{k}\left(X^{X}, \mathrm{id}_{X}\right) \rightarrow \pi_{k}(X)\right)$.

The Gottlieb groups are not natural with respect to induced maps. However, if $X$ is 1-connected and $p_{m}: X \rightarrow X_{m}$ is the $m$-equivalence to the $m$ 'th stage of the Postnikov tower for $X$ then due to [4, Lemma 7] $p_{m *} G_{k}(X) \subseteq G_{k}\left(X_{m}\right)$ for all $k, m$. In particular, $q_{m *} G_{k}\left(X_{m}\right) \subseteq G_{k}\left(X_{m-1}\right)$, where $q_{m}: X_{m} \rightarrow X_{m-1}$ is a principal $K\left(\pi_{m}(X), m\right)$-fibration from the Postnikov tower for $X$.

By the Künneth formula and the fact that $H^{*}\left(\mathbb{S}^{k}, \mathbb{Z}\right)$ has no torsion we have $H^{*}\left(X \times \mathbb{S}^{k}, \pi\right)=H^{*}(X, \pi) \otimes H^{*}\left(\mathbb{S}^{k}, \mathbb{Z}\right)$ for any Abelian group $\pi$. Thus, if $F: \mathbb{S}^{k} \times X \rightarrow X$ is an associated map for $\alpha: \mathbb{S}^{k} \rightarrow X$, then for each $x \in H^{n}(X, \pi)$ the induced map on cohomology gives

$$
F^{*}(x)=1 \otimes x+\lambda_{k} \otimes x_{F}
$$

for some $x_{F} \in H^{n-k}(X, \pi)$, where $\lambda_{k}$ is a chosen generator of $H^{k}\left(\mathbb{S}^{k}, \mathbb{Z}\right)$. Although $x_{F}$ depends on $\lambda_{k}$ we do not denote this. Let $K(\pi, r)$ be the Eilenberg-MacLane space of type $(\pi, r)$ for $r>0$ and $\iota$ the fundamental class in $H^{r+1}(K(\pi, r+1), \pi)$. For a principal $K(\pi, r)$-fibration $p: E \rightarrow B$ via a map $\eta: B \rightarrow K(\pi, r+1)$ let $\mu=\eta^{*}(\iota)$ be an element in $H^{r+1}(B, \pi)$. The following theorem was proved in [3] (Theorem 6-3) and in [7] (Theorem $1)$.

Theorem 1.1. Let $p: E \rightarrow B$ be a principal $K(\pi, r)$-fibration for $r>0$ and let $F: \mathbb{S}^{k} \times B \rightarrow B$ be a map with $\left.F\right|_{B}=\operatorname{id}_{B}$ and $F^{*}(\mu)=1 \otimes \mu+\lambda_{k} \otimes \mu_{F}$. Then there exists a lifting $\widetilde{F}: \mathbb{S}^{k} \times E \rightarrow E$ with $\left.\widetilde{F}\right|_{E}=\operatorname{id}_{E}$ and a homotopy commutative diagram



if and only if $\mu_{F}=0$ in $H^{r+1-k}(B, \pi)$.

Given a finite Abelian group $\pi$, let $l(\pi)$ denote the minimum positive integer such that $l(\pi) \pi=0$.

Proposition 1.2. Let $E \rightarrow B$ be a principal $K(\pi, n)$-fibration for a finite, Abelian group $\pi$ and $F: \mathbb{S}^{k} \times B \rightarrow B$ an extension of $\alpha \vee \operatorname{id}_{B}: \mathbb{S}^{k} \vee B \rightarrow B$. Then the map $\alpha l(\pi) \vee \operatorname{id}_{B}: \mathbb{S}^{k} \vee E \rightarrow E$ has an extension to $\mathbb{S}^{k} \times E$ which is a lifting of the map $F \circ\left(l(\pi) \times \operatorname{id}_{B}\right)$, where $l(\pi)$ is identified with a self map of $\mathbb{S}^{k}$ with degree $l(\pi)$.

Proof. Consider the composite map $F_{1}=F \circ\left(l(\pi) \times \mathrm{id}_{B}\right): \mathbb{S}^{k} \times B \rightarrow B$. With respect to this new map $F_{1}$ the obstruction for lifting is $\mu_{F_{1}}=l(\pi) \mu_{F}$. Since 
multiplication by $l(\pi)$ in the coefficient group $\pi$ is the trivial homomorphism the result follows from Theorem 1.1.

Let $\mathrm{X}$ be a $C W$-space such that homology groups

$$
H_{k}(X, \mathbb{Z})=0, \quad k>n
$$

or homotopy groups

$$
\pi_{k}(X)=0, \quad k>n
$$

and the canonical map

$$
X \rightarrow K\left(\pi_{1}(X), 1\right) \text { is simple, }
$$

where $n$ is sufficiently large. Then, according to [9, Chap. VIII, Theorem 6] the map $X \rightarrow K\left(\pi_{1}(X), 1\right)$ admits a Moore-Postnikov tower. Thus we can state the main result of this section.

Theorem 1.3. Let a space $X$ satisfies (iii) and either (i) or (ii). For a fixed integer $k>1$, suppose that the homotopy groups $\pi_{k+i}(X)$ are finite for $i=1, \ldots, n+k-1$ and $n$ as in (i), (ii), (iii) above. Then $l \pi_{k}(X) \subseteq G_{k}(X)$, where $l=l\left(\pi_{k+1}(X)\right) \cdots l\left(\pi_{n+k-1}(X)\right)$.

Proof. Let $X_{m}$ be the $m$ 'th stage of the Moore-Postnikov tower for the map $f: X \rightarrow K\left(\pi_{1}(X), 1\right)$ with $m \geq 1$. For any map $\alpha: \mathbb{S}^{k} \rightarrow X$ consider the composite $\mathbb{S}^{k} \stackrel{\alpha}{\rightarrow} X \rightarrow X_{k-1}$. Then we certainly have an associated map $F_{k-1}: \mathbb{S}^{k} \times X_{k-1} \rightarrow X_{k-1}$ being in fact the projection. The obstruction $\mu_{F_{k-1}}$ stated in Theorem 1.1 vanishes, so we have a lifting $\mathbb{S}^{k} \times X_{k} \rightarrow X_{k}$ of the map $F_{k-1}$. Now, in light of Proposition 1.2 and inductive arguments, we have a map $\mathbb{S}^{k} \times X_{n+k-1} \rightarrow X_{n+k-1}$ associated to the map $\alpha l\left(\pi_{k+1}(X)\right) \cdots l\left(\pi_{n+k-1}(X)\right)$. The properties of the space $X$ imply that this map lifts to $\mathbb{S}^{k} \times X \rightarrow X$ and the result follows.

In particular, we derive an extension of the Pak and Woo estimate proved in [8] for finite cyclic groups only.

Corollary 1.4. If $X / H$ is the orbit space of any free action of a finite group $H$ on a homotopy $(2 n-1)$-sphere $X$ then $l \mathbb{Z} \subseteq G_{2 n-1}(X / H)$, where $l=l\left(\pi_{2 n}(X)\right) \cdots l\left(\pi_{4 n-3}(X)\right)$.

The proof follows directly from Theorem 1.3 since the homotopy groups in question are finite and $\pi_{2 n-1}(X / H)=\pi_{2 n-1}(X)=\mathbb{Z}$.

\section{Gottlieb groups of orbit spaces.}

Let $X$ be a 1-connected space $X$ of finite type and a finite group $H$

(iv) acting freely on $X$ by homeomorphisms homotopic to the identity.

Then, due to [3, Theorem 6-2] $\eta_{*} G_{k}(X) \supseteq G_{k}(X / H)$ for $k>1$, where $\eta: X \rightarrow X / H$ is the quotient map. In order to search for the converse 
inclusion we need some general facts. Let $\eta: \tilde{Y} \rightarrow Y$ be a universal covering of a space $Y$ of finite type with $\pi_{1}(Y)=H$ being finite. Write $\mathbb{Z} / p$ for the cyclic group of order $p$ and $\mathbb{Q}$ for the rational numbers. Then the following holds.

Proposition 2.1. The map $\eta: \tilde{Y} \rightarrow Y$ induces a cohomology and homology isomorphism with coefficients in either $\mathbb{Q}$ or $\mathbb{Z} / p$ provided that $p$ is relatively prime to the order $|H|$ of the group $H$. The kernel of the induced map $\eta^{*}: H^{*}(Y, \mathbb{Z}) \rightarrow H^{*}(\tilde{Y}, \mathbb{Z})$ consists only of torsion elements the order of which has prime factorizations containing only primes dividing the order $|H|$ of the group $H$.

Proof. Consider the Serre spectral sequence associated with the fibration $\tilde{Y} \rightarrow Y \rightarrow K(H, 1)$. We know that the term $E_{2}^{i, j}$ of this spectral sequence is given by $H^{i}\left(K(H, 1), H^{j}(\tilde{Y}, \mathbb{Q})\right)$. Since the coefficient group $H^{j}(\tilde{Y}, \mathbb{Q})$ is a direct sum of the rationals $\mathbb{Q}$, the action of the base in the cohomology of the fibre is trivial and the group $H$ is finite, it follows that $E_{2}^{i, j}=0$ for $i>0$. But $H^{0}\left(K(H, 1), H^{j}(\tilde{Y}, \mathbb{Q})\right)=H^{j}(\tilde{Y}, \mathbb{Q})$ and consequently the result follows for coefficients in $\mathbb{Q}$. In the same way we can also show that result for coefficients in $\mathbb{Z} / p$ provided $p$ is relatively prime to the order $|H|$ of the group $H$.

The space $Y$ is of finite type, so the above cohomology isomorphisms induce homology isomorphisms with the same coefficients as well. Thus we get that the induced map $\bar{\eta}_{*}: H_{*}(Y, \mathbb{Z}) / T\left(H_{*}(Y, \mathbb{Z})\right) \rightarrow H_{*}(\tilde{Y}, \mathbb{Z}) / T\left(H_{*}(\tilde{Y}, \mathbb{Z})\right)$ (modulo the torsion parts) is a monomorphism with a finite cokernel. Therefore, the induced map $\operatorname{Hom}\left(H_{*}(Y, \mathbb{Z}), \mathbb{Z}\right) \rightarrow \operatorname{Hom}\left(H_{*}(\tilde{Y}, \mathbb{Z}), \mathbb{Z}\right)$ is a monomorphism. Moreover, we can deduce that for any positive integer $n$ the map $\eta: \tilde{Y} \rightarrow Y$ also induces cohomology and homology isomorphisms with coefficients in the cyclic groups $\mathbb{Z} / p^{n}$.

For any $k>1$, from the commutative diagram



determined by the Universal Coefficient Theorem, it follows that the kernel of the induced map $\eta^{*}: H^{*}(Y, \mathbb{Z}) \rightarrow H^{*}(\tilde{Y}, \mathbb{Z})$ is contained in the group $\operatorname{Ext}\left(H_{k-1}(Y, \mathbb{Z}), \mathbb{Z}\right)$ being the torsion part of the group $H^{k}(Y, \mathbb{Z})$. If $T_{H}\left(H_{k-1}(Y, \mathbb{Z})\right)$ and $T_{H}\left(H_{k-1}(\tilde{Y}, \mathbb{Z})\right)$ are the subgroups of $H_{k-1}(Y, \mathbb{Z})$ and $H_{k-1}(\tilde{Y}, \mathbb{Z})$, respectively consisting of all elements with the order relatively prime to the order $|H|$ of the group $H$ and $p$ is a prime not dividing the $|H|$ then the induced isomorphism $\operatorname{Ext}\left(H_{k-1}(Y, \mathbb{Z}), \mathbb{Z} / p^{n}\right) \rightarrow$ 
$\operatorname{Ext}\left(H_{k-1}(\tilde{Y}, \mathbb{Z}), \mathbb{Z} / p^{n}\right)$ restricts to the $\operatorname{map} T_{H}\left(H_{k-1}(Y, \mathbb{Z})\right) \otimes \mathbb{Z} / p^{n} \rightarrow$ $T_{H}\left(H_{k-1}(\tilde{Y}, \mathbb{Z})\right) \otimes \mathbb{Z} / p^{n}$ for all positive integers $n \geq 1$. Consequently, the induced map $T_{H}\left(H_{k-1}(\tilde{Y}, \mathbb{Z})\right) \rightarrow T_{H}\left(H_{k-1}(Y, \mathbb{Z})\right)$ is an isomorphism as well.

Let $T_{H}^{\prime}\left(H_{k-1}(Y, \mathbb{Z})\right)$ be the subgroup of $H_{k-1}(Y, \mathbb{Z})$ consisting of all elements with the order non-relatively prime to the order $|H|$ of the group $H$. We get that the kernel of the induced map $\eta^{k}: H^{k}(Y, \mathbb{Z}) \rightarrow H^{k}(\tilde{Y}, \mathbb{Z})$ is contained in the group $\operatorname{Ext}\left(H_{k-1}(Y, \mathbb{Z}), \mathbb{Z}\right)=T_{H}^{\prime}\left(H_{k-1}(Y, \mathbb{Z})\right)$ and the proof is complete.

Let now $X$ be a space with a free $H$-action and $\eta: X \rightarrow X / H$ the quotient map. Then the induced maps $\eta_{m}: X_{m} \rightarrow(X / H)_{m}$ of the $m$ 'th Postnikov stages are coverings with $\pi_{1}\left((X / H)_{m}\right)=H$ for all $m \geq 1$. Therefore, Proposition 2.1 yields:

Corollary 2.2. If $X$ is a 1-connected space of finite type with a free $H$ action satisfying (iv) and $\eta: X \rightarrow X / H$ the quotient map then the induced maps $\eta_{m}: X_{m} \rightarrow(X / H)_{m}$ of the $m$ 'th Postnikov stages are cohomology and homology isomorphisms with coefficients in $\mathbb{Q}$ or $\mathbb{Z} / p$ provided that $p$ is relatively prime with the order $|H|$ of the $H$ for all $m \geq 1$. The kernel of the induced maps $H^{*}\left((X / H)_{m}, \mathbb{Z}\right) \rightarrow H^{*}\left(X_{m}, \mathbb{Z}\right)$ consists of only torsion elements the order of which have prime factorization containing only primes dividing the order $|H|$ of the group $H$.

Theorem 2.3. Let $X$ be a 1-connected space of finite type satisfying either (i) or (ii) and with a free $H$-action satisfying (iv). Then:

(1) if $p$ is a prime not dividing the order $|H|$ of the group $H$ then $\eta_{*} G_{k}(X)_{p}$ $=G_{k}(X / H)_{p}$ for the p-primary components;

(2) for each $k>1$ there is a positive integer $l_{k}$ such that $l_{k} \eta_{*} G_{k}(X) \subseteq$ $G_{k}(X / H)$.

Proof. (1). Let $\alpha$ be an element in the $p$-primary component $G_{k}(X)_{p}$ of the Gottlieb group $G_{k}(X)$ for $k>1$. Suppose we have found a lifting $F: \mathbb{S}^{k} \times(X / H)_{m} \rightarrow(X / H)_{m}$ to the $m^{\prime}$ th stage of the Postnikov tower of the space $X / H$ for the composite $\mathbb{S}^{k} \stackrel{\alpha}{\rightarrow} X \rightarrow X / H \rightarrow(X / H)_{m}$. In order to find a lifting to the next stage we compute $\mu_{F}$ which is a cohomology class in $H^{m+1-k}\left((X / H)_{m}, \pi\right)$ with coefficients in the group $\pi=\pi_{m+1}(X)=$ $\pi_{m+1}(X / H)$. The space $X$ is of finite type so the group $\pi$ is a direct sum of its free part, a torsion part consisting of all elements which have order relatively prime to the order $|H|$ of the group $H$ and those torsion elements whese orders have prime factors dividing $|H|$. To compare the obstruction for liftings (of an associated map) from the Postnikov tower of $X$ to that tower of its quotient $X / H$ we observe:

a) If the group $\pi$ is finite with torsion relatively prime to the order $|H|$ of the group $H$, then by Proposition 2.1 we have an isomorphism $H^{*}\left((X / H)_{m}, \pi\right) \rightarrow H^{*}\left(X_{m}, \pi\right)$. 
b) If $\pi$ is a torsion group, where the order of any of its elements is a product of primes which divide the order $|H|$ of the group $H$ then $\mu_{F}=0$. For, let $q$ be a number relatively prime to the order $|H|$ of the group $H$ and such that $q \alpha=0$. Then by Theorem 1.1 we get $q \mu_{F}=0$. But the multiplication by $q$ is an isomorphism of the group $\pi$ and the conclusion follows.

c) If the coefficient is the integers $\mathbb{Z}$ then by Corollary 2.2 we have that $\mu_{F}$ is a torsion element and the prime factorization of its order has only primes dividing the order $|H|$ of the group $H$. Thus, by the same argument as in b), we get that $\mu_{F}=0$.

These three observations together tell us that $\mu_{F}=0$ and, in the light of Theorem 1.1, the first part is proved.

(2). It is enough to observe that for each Postnikov stage $(X / H)_{m}$, the obstruction $\mu_{F}$ has finite order. This follows from the analysis given above together with the fact that we start with an element in $G_{k}(X)$. Since we need to go through only a finite number of stages in the Postnikov tower of the space $X / H$ the result follows.

Remark 2.4. The numbers $l$ given in the theorem above can sometimes be estimated. Suppose $H_{i}(X, \mathbb{Z})=0$ or $\pi_{i}(X)=0$ for $i \geq n$ and let conn $X$ be the connectivity of the space $X$. Then, to show the existence of a positive integer $l$ with $l \eta_{*} G_{k}(X) \subseteq G_{k}(X / H)$, it is sufficient to consider only the stages $(X / H)_{m}$ for $k+\operatorname{conn} X-1 \leq m \leq k+n-1$ in the Postnikov tower for the space $X / H$. It follows from the proof of Theorem 2.3 that the prime factorization of the integer $l$ contains only the primes $p$ which divide $|H|$, the order of the group $H$ and at least one of the homotopy groups $\pi_{m}(X)$ has a nontrivial $p$-primary component for $k+\operatorname{conn} X-1 \leq m \leq k+n+1$.

\section{Examples and computations.}

In this section we give some examples of applications of results or methods of the previous sections to calculate or estimate some Gottlieb groups.

Example 3.1. Let $X$ be a homotopy $(2 n-1)$-sphere with a free $H$-action of a finite group $H$. From the Lefschetz fixed point theorem it follows that this action is orientable. We claim the following:

(1) For every odd prime $p$ (not dividing the order $|H|$ of the group $H$ ) the $p$-primary component of the Gottlieb group $G_{k}(X / H)$ is equal to the $p$-primary component of the homotopy group $\pi_{k}(X)$;

(2) The Gottlieb group $G_{2 n-1}(X / H)$ is nontrivial and there is a positive integer $l$ such that $l \mathbb{Z} \subseteq G_{2 n-1}(X / H)$. If $n=2$ then the number $l=4$ satisfies this condition.

Proof. (1). By Theorem 2.3 we have that the $p$-primary components of the $k^{\prime}$ th Gottlieb groups of the spaces $X$ and $X / H$ are isomorphic for $k>1$. 
In view of [1] an odd dimensional homotopy sphere localized at an odd prime $p$ is an $H$-space so we get in light of [6, Corollary 2.5] that the $p$ primary component of the Gottlieb group $G_{k}(X / H)$ is isomorphic to the $k^{\prime}$ th homotopy group $\pi_{k}\left(X_{p}\right)$ of the $p$-localization $X_{p}$ of the space $X$ and the result follows.

(2) The inclusion $l \mathbb{Z} \subseteq G_{2 n-1}(X / H)$, for some positive integer $l$, is shown in Corollary 1.4. If $n=2$ then to estimate the number $l$ we have to construct the lifting maps up to the 5'th stage of the Postnikov tower for the space $X / H$. Of course, there is no obstruction at the third stage. So we use principal $K\left(\pi_{4}\left(\mathbb{S}^{3}\right), 5\right)$ - and $K\left(\pi_{5}\left(\mathbb{S}^{3}\right), 6\right)$-fibrations only. But $\pi_{4}\left(\mathbb{S}^{3}\right)=\pi_{5}\left(\mathbb{S}^{3}\right)=\mathbb{Z} / 2$ and the result follows.

Example 3.2. (1) Consider an even dimensional sphere $\mathbb{S}^{2 n}$. Gottlieb in $\left[3\right.$, Theorem 5-4] proved that $G_{2 n}\left(\mathbb{S}^{2 n}\right)=0$. We claim that the Gottlieb group $G_{4 n-1}\left(\mathbb{S}^{2 n}\right)$ is nontrivial and contains a subgroup of rank one.

(2) $G_{k}\left(\mathbb{S}^{2}\right)=\pi_{k}\left(\mathbb{S}^{2}\right)$ for all $k \geq 3$.

Proof. (1). In view of Theorem 1.3 it suffices to observe that the homotopy groups $\pi_{4 n}\left(\mathbb{S}^{2 n}\right), \ldots, \pi_{6 n-2}\left(\mathbb{S}^{2 n}\right)$ are finite. So the result follows.

(2). Consider the long exact sequence for the Hopf fibration $\mathbb{S}^{1} \rightarrow \mathbb{S}^{3} \stackrel{p}{\rightarrow}$ $\mathbb{S}^{3} / \mathbb{S}^{1}=\mathbb{S}^{2}$

$$
\cdots \rightarrow \pi_{n}\left(\mathbb{S}^{3}\right) \stackrel{p_{*}}{\rightarrow} \pi_{n}\left(\mathbb{S}^{2}\right) \rightarrow \pi_{n-1}\left(\mathbb{S}^{1}\right) \rightarrow \cdots .
$$

But $\pi_{k-1}\left(\mathbb{S}^{1}\right)=0$ for $k \geq 3$. Then by exactness $p_{*} \pi_{k}\left(\mathbb{S}^{3}\right)=\pi_{k}\left(\mathbb{S}^{2}\right)$ for $k \geq 3$. On the other hand, by [5, Theorem II.5] $p_{*} \pi_{k}\left(\mathbb{S}^{3}\right) \subseteq G_{k}\left(\mathbb{S}^{2}\right)$ for all $k \geq 1$ and the proof is complete.

Let $L_{2 n+1}(p)$ be a $(2 n+1)$-dimensional lens space. Then Pak and Woo have shown in $[8]$ that

$$
G_{2 n+1}\left(L_{2 n+1}(p)\right)= \begin{cases}\mathbb{Z}, & \text { if } n=0,1,3, \\ 2 \mathbb{Z}, & \text { for any other } n .\end{cases}
$$

Remark 3.3. (1) Let $H \subseteq S O(2 n+2)$ be a finite subgroup of the special orthogonal group $S O(2 n+2)$ acting freely on a $(2 n+1)$-dimensional sphere $\mathbb{S}^{2 n+1}$. Those groups have been fully classified by Wolf in [11]. Consider the fibration

$$
\mathbb{S}^{2 n+1} / H \rightarrow V_{2 n+3,2} / H \rightarrow \mathbb{S}^{2 n+2},
$$

where $V_{2 n+3,2}$ is the Stiefel manifold which may be interpreted as the space of all unit vectors tangent to the sphere $\mathbb{S}^{2 n+1}$. Then, following mutatis mutandis, the methods presented by Pak and Woo in [8], we also obtain that

$$
G_{2 n+1}\left(\mathbb{S}^{2 n+1} / H\right)= \begin{cases}\mathbb{Z}, & \text { if } n=0,1,3, \\ 2 \mathbb{Z}, & \text { for any other } n .\end{cases}
$$

(2) Observe that the Postnikov tower of the rationalization $\mathbb{S}_{0}^{2 n}$ of an even dimensional sphere $\mathbb{S}^{2 n}$ is given by the diagram 


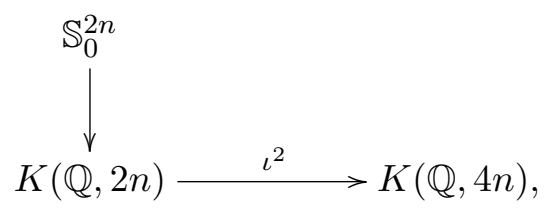

where $\iota$ is the fundamental class in $H^{2 n}(K(\mathbb{Q}, 2 n), \mathbb{Q})$ and the map $\mathbb{S}_{0}^{2 n} \rightarrow$ $K(\mathbb{Q}, 2 n)$ is a principal $K(\mathbb{Q}, 4 n-1)$-fibration via the map $\iota^{2}: K(\mathbb{Q}, 2 n) \rightarrow$ $K(\mathbb{Q}, 4 n)$.

Assume that $F: \mathbb{S}^{2 n} \times \mathbb{S}_{0}^{2 n} \rightarrow \mathbb{S}_{0}^{2 n}$ is an associated map for $\alpha$ in the group $\pi_{2 n}\left(\mathbb{S}_{0}^{2 n}\right)$. If $u: \mathbb{S}_{0}^{2 n} \rightarrow K(\mathbb{Q}, 2 n)$ is a generator of the cohomology group $H^{2 n}\left(\mathbb{S}_{0}^{2 n}, \mathbb{Q}\right)=\mathbb{Q}$ then as in $\left[\mathbf{5}\right.$, Theorem III.6] we get that $\alpha^{*} u=0$. Thus $\alpha$ is null homotopic and by [6, Corollary 2.5] we obtain that $G_{2 n}\left(\mathbb{S}_{0}^{2 n}\right)=$ $G_{2 n}\left(\mathbb{S}^{2 n}\right)_{0}=0$. Consequently, we have reproved the Gottlieb result from [3, Theorem 5-4] that $G_{2 n}\left(\mathbb{S}^{2 n}\right)=0$ since $\pi_{2 n}\left(\mathbb{S}^{2 n}\right)=\mathbb{Z}$.

Remark 3.4. Let now $\mathbb{C} P^{n}, \mathbb{H} P^{n}$ be the complex and quaternion projective spaces, respectively for $n \geq 1$ and $\mathbb{C} a P^{2}$ the Cayley projective plane. Then the rationalization of the Hopf fibrations $\mathbb{S}^{2 n+1} \rightarrow \mathbb{C} P^{n}$ and $\mathbb{S}^{4 n+3} \rightarrow \mathbb{H} P^{n}$ yields that the homotopy groups of the rationalizations $\mathbb{C} P_{0}^{n}$ and $\mathbb{H} P_{0}^{n}$ of the projective spaces $\mathbb{C} P^{n}$ and $\mathbb{H} P^{n}$, respectively are given as follows:

$$
\begin{aligned}
& \pi_{k}\left(\mathbb{C} P_{0}^{n}\right)= \begin{cases}\mathbb{Q}, & \text { if } k=2,2 n+1, \\
0, & \text { for any other } k\end{cases} \\
& \pi_{k}\left(\mathbb{H} P_{0}^{n}\right)= \begin{cases}\mathbb{Q}, & \text { if } k=4,4 n+3, \\
0, & \text { for any other } k .\end{cases}
\end{aligned}
$$

To compute the rational homotopy groups of the space $\mathbb{C} a P^{2}$ consider the fundamental class $\iota_{8}$ in $H^{8}(K(\mathbb{Q}, 8), \mathbb{Q})$ and the principal $K(\mathbb{Q}, 23)$-fibration $E \rightarrow K(\mathbb{Q}, 8)$ via the map $\iota_{8}^{3}: K(\mathbb{Q}, 8) \rightarrow K(\mathbb{Q}, 24)$. If $u: \mathbb{C} a P^{2} \rightarrow$ $K(\mathbb{Q}, 8)$ is the generator of the cohomology group $H^{8}\left(\mathbb{C} a P^{2}, \mathbb{Q}\right)=\mathbb{Q}$ then $\left(\iota_{8}^{3}\right)^{*} u=0$. Therefore, there is a map $\varphi: \mathbb{C} a P^{2} \rightarrow E$ and it is easy to check that the induced map $\varphi^{*}: H^{*}(E, \mathbb{Q}) \rightarrow H^{*}\left(\mathbb{C} a P^{2}, \mathbb{Q}\right)$ in cohomology is an isomorphism. Consequently, the space $\mathbb{C} a P^{2}$ has the rational homotopy type of the space $E$ and we get

$$
\pi_{k}\left(\mathbb{C} a P_{0}^{2}\right)= \begin{cases}\mathbb{Q}, & \text { if } k=8,23, \\ 0, & \text { for any other } k .\end{cases}
$$

Postnikov towers of the rational spaces $\mathbb{C} P_{0}^{n}, \mathbb{H} P_{0}^{n}$ and $\mathbb{C} a P_{0}^{2}$ are determined by the invariants $\iota_{2}^{n+1}: K(\mathbb{Q}, 2) \rightarrow K(\mathbb{Q}, 2 n+2), \iota_{4}^{n+1}: K(\mathbb{Q}, 4) \rightarrow$ $K(\mathbb{Q}, 4 n+4)$ and $\iota_{8}^{3}: K(\mathbb{Q}, 8) \rightarrow K(\mathbb{Q}, 24)$, where $\iota_{2}, \iota_{4}$ and $\iota_{8}$ are the fundamental classes in $H^{2}(K(\mathbb{Q}, 2), \mathbb{Q}), H^{4}(K(\mathbb{Q}, 4), \mathbb{Q})$ and $H^{8}(K(\mathbb{Q}, 8), \mathbb{Q})$, respectively. Following the methods presented above for spheres of even dimension, we can state that $G_{2}\left(\mathbb{C} P^{n}\right)=G_{4}\left(\mathbb{H} P^{n}\right)=G_{8}\left(\mathbb{C} a P^{2}\right)=0$ since 
$\pi_{2}\left(\mathbb{C} P^{n}\right)=\pi_{4}\left(\mathbb{H} P^{n}\right)=\pi_{8}\left(\mathbb{C} a P^{2}\right)=\mathbb{Z}$ for all $n \geq 1$. In particular, the Lang result [5, Theorem III.6] has been reproved.

\section{References}

[1] J.F. Adams, The sphere considered as an H-space mod-p, Quart. J. Math., 12 (1961), $52-60$.

[2] D. Gottlieb, A certain subgroup of the fundamental group, Amer. J. of Math., 87 (1965), 840-856.

[3] _ Evaluation subgroups of homotopy groups, Amer. J. of Math., 91 (1969), 729-756.

[4] H.B. Haslam, $G$-spaces mod $F$ and $H$-spaces mod $F$, Duke Math. J., 38 (1971), 671-679.

[5] G.E. Lang, Evaluation subgroups of factor spaces, Pac. J. Math., 42(3) (1972), 701709 .

[6] , Localizations and evaluation subgroups, Proc. Amer. Math. Soc., 5 (1975), 489-494.

[7] J. Oprea, Finite group actions on spheres and the Gottlieb group, J. Korean Math. Soc., 1 (1991), 65-78.

[8] J. Pak and M.H. Woo, Gottlieb groups of lens spaces, Bull. Korean Math. Soc., 36(3) (1999), 621-627.

[9] E. Spanier, Algebraic Topology, McGraw-Hill (1966).

[10] N.E. Steenrod and D.B.A. Epstein, Cohomology Operations, Ann. of Math. Studies, 50, Princeton (1962).

[11] J.A. Wolf, Spaces of Constant Curvature, New-York, McGraw-Hill (1967).

Received August 7, 1998 and revised January 20, 2000. This work was initiated during the visit of the first author to the Departamento de Matemática - USP - Brasil, August 1998. This visit has been supported by FAPESP-São Paulo-Brasil.

Faculty of Mathematics and Informatics

Nicholas Copernicus University

Chopina 12/18, 87-100 ToRuń

POLAND

E-mail address: marek@mat.uni.torun.pl

Department of Mathematics-IME

University of São Paulo

Caixa Postal 66.281-AG

05315-970 SÃo PAULO

BRASIL

E-mail address: dlgoncal@ime.usp.br 